\title{
Gestión sostenible del paisaje visual en el término municipal de Rascafría (Comunidad de Madrid)
}

\author{
Elena Bronchalo González
}

RESUMEN

La evolución que la sociedad ha experimentado en las últimas décadas, caracterizada por un fuerte desarrollo económico, ha llevado a hacer uso del

Paisaje como Recurso Natural que satisfaga sus necesidades. En este momento podremos hablar de su Gestión Sostenible al hacer referencia a cualquier actividad que pueda modificarlo artificialmente. Mediante una serie de Criterios e Indicadores se analiza la Sostenibilidad del Paisaje Visual para valorar el Riesgo de Pérdida de la misma en las distintas Unidades de Paisaje en la que se puede dividir el territorio.

PALABRAS CLAVE:

Sostenibilidad del Paisaje, Gestión Sostenible del Paisaje Visual, Criterios e Indicadores, Riesgo de Pérdida de Sostenibilidad, Unidad de Paisaje.

Desarrollo sostenible, Gestión sostenible

Sostenibilidad del Paisaje Visual: Utilización racional de los recursos para aumentar el bienestar de la población, pero cuidando de no agotarlos o destruirlos, de forma que su aprovechamiento pueda ser prolongable en el tiempo. Es decir, un paisaje será sostenible, si los beneficios económicos, sociales y medioambientales que proporciona se mantienen o aumentan con los años.

\section{ABSTRACT}

The evolution that society has gone throught in the last decades, characterized by a severe economic development, has been one of the reasons why nowadays men use the Landscape as a Natural Resource which satisfied its needs. From this moment is when we can start talking about its sustainable management when we make any reference to any kind of activity able to modify it. Using some Criteria and Indicators we will try to analyze the Visual Landscape Sustainability in order to obtain the risk of losing it in the different Landscape units in which we can divide one land.

* Ingeniero de montes. Ayagavres Medioambiente. 


\section{INTRODUCCIÓN}

Los recursos naturales son los elementos básicos o primarios, susceptibles de un aprovechamiento, que proporciona la naturaleza en su estado primitivo. El paisaje es un recurso natural, al igual que el agua, el aire, el suelo etc. En el concepto de recurso está implícita la posibilidad de aprovechamiento por la sociedad. Todos los recursos tienen un valor económico, que está más relacionado con su abundancia o escasez, que con parámetros de otro tipo, como los costes de su aprovechamiento, transporte o con la energía empleada en su utilización o almacenamiento.

La valoración del paisaje natural está en alza en todos los paises y, muy especialmente en Europa. Las altas densidades de población, los cultivos, las industrias y las edificaciones afectan a la calidad del paisaje por sus efectos, tanto directos como indirectos.

En la actualidad, a medida que la sociedad aumenta su nivel de desarrollo y de satisfacción de las necesidades básicas, retorna a usar los recursos que satisfacen sus necesidades sensoriales y espirituales, entre las que ocupa un lugar destacado el paisaje. De esta manera, en los últimos años, se ha convertido en un bien codiciado, que produce beneficios económicos, motivo por el cual es frecuentemente agredido por la intervención humana, al igual que les sucede a los demás recursos naturales.

Frente a las agresiones que el desarrollo produce en los recursos naturales han surgido diversas posturas. En un extremo se encuentran las conservacionistas, defensoras del mantenimiento de la naturaleza en estado puro, y en el otro, las posturas desarrollistas. que ponen el bienestar material de la población por encima de la conservación de los recursos. La evolución de ambas posturas converge en el concepto de Sostenibilidad, es decir, la utilización racional de los recursos para aumentar el bienestar de la población, pero cuidando de no agotarlos o destruirlos, de forma que su aprovechamiento pueda mantenerse, a fin de que puedan ser aprovechados por las generaciones futuras.

En un primer momento se utilizó el término desarrollo sostenible para designar con él todas las actividades que conducian a una mejora de la población, teniendo en cuenta la disponibilidad, presente y futura, de los recursos naturales. En poco tiempo se generalizó el término a todas las actividades, incluso a las que no necesariamente producen un desarrollo inmediato de la población afectada, tendentes a asegurar la permanencia de estos recursos en el tiempo, denominándose entonces gestión sostenible. Este concepto es aplicable a todos los recursos naturales, incluido el paisaje. Sin embargo, en este caso, la diferenciación entre desarrollo y gestión no aparece clara, al contrario que en el resto de los recursos.

El paisaje, por su propia naturaleza, es dinámico y cambiante, incluso sin intervención humana, por lo que parece más adecuado utilizar el concepto de gestión al hablar de cualquier actuación que pueda modificarlo artificialmente. 
En este artículo resumimos un trabajo mucho más amplio que constituyó el proyecto fin de carrera titulado "Gestión Sostenible del Paisaje Visual. Criterios e Indicadores. Aplicación a la Comunidad de Madrid», en el que se muestra un método para evaluar el riesgo de pérdida de sostenibilidad del paisaje visual.

\section{ÁREA DE ESTUDIO}

El ámbito territorial de estudio comprende diez Unidades de Paisaje de la Comunidad de Madrid, ubicadas en el término municipal de Rascafría y en los términos limítrofes.

Por Unidad de Paisaje entendemos una fracción de la superficie que presente una respuesta visual homogénea, tanto en sus componentes paisajísticos, como en su respuesta visual ante posibles actuaciones. En la Comunidad de Madrid, la Unidad Docente de Planificación y Proyectos de la E.T.S.I. Montes, definió 237 unidades de paisaje.

El mérmino municipal de Rascafría se encuentra enclavado en la parte más occidental del Valle Alto del Lozoya, en la parte noroccidental de la Comunidad de Madrid, en la Sierra de Guadarrama. El valle, situado a unos 1.100 metros de altitud se encuentra delimitado por dos cadenas montañosas: la septentrional, divisoria entre Madrid y Segovia, en la margen izquierda del Lozoya. Esto se extiende desde Cotos al Portachuelo de Navarredonda, con altitudes que oscilan entre 1.800 y $2.200 \mathrm{~m}$. Los pasos a Segovia se producen por los Puertos de Cotos, EI Reventón, Malgosto y Navafría. Su límite sur discurre por la Cuerda Larga, en donde se elevan las cumbres de Guarramillas, Valdemartín y Cabeza de Hierro

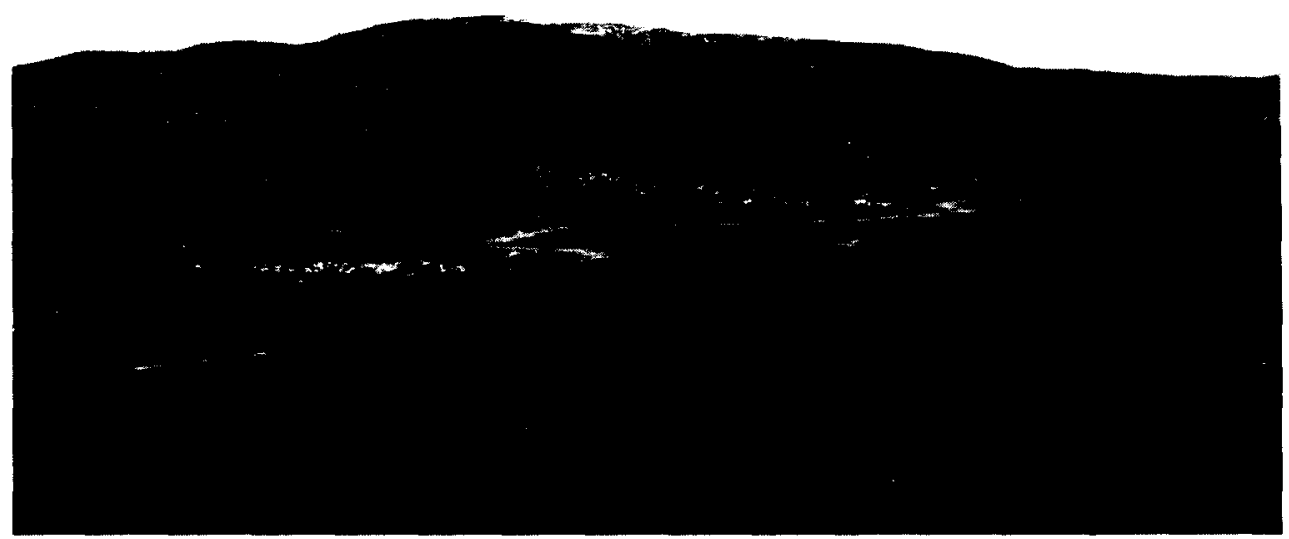

Figura 1. Término municipal de Rascafría desde El Puerto de la Morcuera. 
$(2.383 \mathrm{~m})$. Al norte de estas cumbres nace el río de la Angostura, que más tarde toma el nombre de Lozoya.

El término municipal de Rascafria, con 150,30 kilómetros cuadrados, es el de mayor extensión de la zona y comprende dos núcleos de población, Rascafría, que es la cabecera municipal, y Oteruelo del Valle. Así mismo, a sólo dos kilómetros del casco de Rascafría, se encuentra El Monasterio de El Paular. El territorio, presenta un alto valor paisajístico, encontrándose poblado de espesos bosques de robles y pinares, que alternan con prados. La fauna es rica y variada.

\section{OBJETIVOS Y PLANTEAMIENTO GENERAL}

El Paisaje, como se ha dicho anteriormente, es un recurso natural y como tal ha de ser preservado. La ausencia de estudios del paisaje que tengan en consideración la sostenibilidad de una zona lleva a plantear la necesidad de enfocar el trabajo hacia este campo con el fin de analizar la sostenibilidad del paisaje visual mediante la aplicación de una serie de criterios e indicadores. De esta manera se contribuye a asegurar su protección, conservación y mejora, asi como al establecimiento de un marco de referencia para las posibles actuaciones encaminadas a la utilización racional del recurso paisaje.

Objetivos:

- Aplicar un modelo general, que permita evaluar la Sostenibilidad del paisaje visual mediante la aplicación de Criterios e Indicadores a la Sierra noroccidental de la Comunidad de Madrid.

- Elaborar una cartografía de Riesgo de Pérdida de Sostenibilidad Visual (probabilidad de que se produzcan cambios en los atributos que definen la calidad o fragilidad visual) de las unidades de paisaje, para analizar, si los cambios que se producen en el paisaje visual, debidos a la actuación de las generaciones presentes para satisfacer sus necesidades, está comprometiendo el disfrute y uso de las generaciones futuras, así como para que sirva de base para la gestión del territorio.

\section{ASPECTOS METODOLÓGICOS}

El método seguido para cumplir los objetivos propuestos, ha consistido, en primer lugar, en la selección de las Unidades de Paisaje, objeto de estudio, de entre todas las definidas en la Comunidad de Madrid. De esta manera, las unidades elegidas han sido diez, pertenecientes al término municipal de Rascafría, anteriormente citado. Las unidades seleccionadas son: Parameras de la Morcuera, Valle de Rascafría, Puerto de Malagosto, Valle de la Morcuera, Puerto del Reventón, Alto Manzanares, Arroyo de la Angostura, Puerto de la Morcuera Sur, La Najarra y Macizo de Peñalara. 
Estas unidades se dividen, desde el punto de vista de la vegetación, en teselas. La clasificación de estas teselas corresponde al Plan Regional de Estrategia Territorial (PRET 1997). Para poder facilitar y reducir el tiempo de análisis, se ha llevado a cabo una reclasificación de dichas teselas, simplificando las unidades definidas en el mapa de vegetación y usos del suelo de la Comunidad de Madrid (PRET).Para ello se han seguido una serie de criterios que se exponen a continuación:

Cuadro 1. Formaciones vegetales del área de estudio

\begin{tabular}{|c|c|c|}
\hline Código & $\begin{array}{l}\text { Formación } \\
\text { Vegetal }\end{array}$ & Clases \\
\hline \multirow{5}{*}{1} & \multirow{5}{*}{ Pastizales } & 1.1. Cervunales y pastizales húmedos \\
\hline & & 1.2. Pastos xerofíticos \\
\hline & & 1.3. Pastos mesófilos no parcelados \\
\hline & & 1.4. Prados reticulares \\
\hline & & 1.5. Lastonares de cumbre \\
\hline \multirow{4}{*}{2} & \multirow{4}{*}{ Matorrales } & 2.1. Jarales \\
\hline & & 2.2. Cantuesares. Tomillares y otros acidófilos de pequeña talla \\
\hline & & $\begin{array}{l}\text { 2.3. Matorrales acidófilos montanos con predominio de legumi- } \\
\text { nosas }\end{array}$ \\
\hline & & 2.4. Piornales y otros matorrales de altura \\
\hline 3 & Roquedos & \\
\hline \multirow{4}{*}{4} & \multirow{4}{*}{ Pinares } & 4.1. Pinares de Pinus sylvestris \\
\hline & & 4.2. Pinares mezcla de Pinus pinaster y Pinus pinea \\
\hline & & 4.3. Pinares mezcla de Pinus sylvestris y Pinus nigra \\
\hline & & 4.4. Pinar mezcla de Pinus pinaster, Pinus sylvestris y Pinus nigra \\
\hline 5 & Enebrales & \\
\hline 6 & $\begin{array}{l}\text { Frondosas } \\
\text { perennifolias }\end{array}$ & Encinares \\
\hline \multirow{4}{*}{7} & \multirow{4}{*}{$\begin{array}{c}\text { Frondosas } \\
\text { caducifolias } \\
y \text { marcescentes }\end{array}$} & 7.1. Quejigares \\
\hline & & 7.2. Melojares \\
\hline & & 7.3. Fresnedas \\
\hline & & $\begin{array}{l}\text { 7.4. Vegetación de ribera (Choperas, olmedas y especies frea- } \\
\text { tofitas) }\end{array}$ \\
\hline 8 & Mezcla & Pinares mezcla de Pinus sylvestris y Quercus pyrenaica \\
\hline 9 & Urbanizados & $\begin{array}{l}\text { Cascos urbanos, urbanizaciones y otros (campings, cementerios, } \\
\text { instalaciones ganaderas..) }\end{array}$ \\
\hline 10 & Embalses & \\
\hline
\end{tabular}


Una vez llevada a cabo esta reclasificación, se pasa a la fase más importante del estudio, que es la de selección y evaluación de los Criterios e Indicadores que se van a utilizar para conseguir el objetivo propuesto de conocer la sostenibilidad del paisaje y el riesgo que éste puede tener de perder tal sostenibilidad.

Se entiende por Criterio, una categoría de condiciones o procesos mediante la cual, la gestión de los espacios naturales será asistida. Estos se caracterizan por los Indicadores, que son la medida de un aspecto del criterio.

\section{Selección de criterios de evaluación}

Se han seleccionado tres criterios, caracterizados a través de cuatro indicadores.

Para su elección se ha buscado información en estudios llevados a cabo sobre sostenibilidad, en los que se usaran criterios e indicadores. En todos ellos, los criterios utilizados resultaron ser similares, lo que variaba de unos a otros eran los indicadores. En nuestro caso, se han seleccionado, de entre todos los posibles, los criterios que pueden ser medidos a través de indicadores visuales.

Desarrollar el conjunto de indicadores para evaluar la sostenibilidad de una Comunidad requiere hacer el balance de muchas necesidades de la misma. En un primer momento, se puede pensar en muchos indicadores, la dificultad vendría a la hora de decidir cuál es el más adecuado. Por este motivo, es muy importante tener abundante información sobre los mismos, así como un buen conocimiento de la zona, ya que esto es útil para su elaboración, desarrollo y aplicación.

En la selección de indicadores, con el fin de lograr una herramienta útil, se trató de que reuniesen una serie de características:ser mensurables, aplicables, significativos, de fácil entendimiento, apropiados en escala, precisos y asequibles económicamente.

\section{Criterio 1. Conservación de la Calidad Visual}

El primero de los criterios es la Conservación de la Calidad Visual, entendiendo por Calidad de un paisaje, el "grado de excelencia de éste, su mérito para no ser alterado o destruido o de otra manera, su mérito para que su esencia y su estructura actual se conserve" (BLANCO, 1979).

El indicador seleccionado para el mismo es el que denominamos "Diversidad visual». La vegetación es uno de los componentes principales del paisaje. Muchas de las características que ésta presenta, como la textura, la variabilidad cromática, debida a la vegetación y a la altitud, la forma, etc., van a tener influencia en la conservación de la sostenibilidad de un paisaje. 


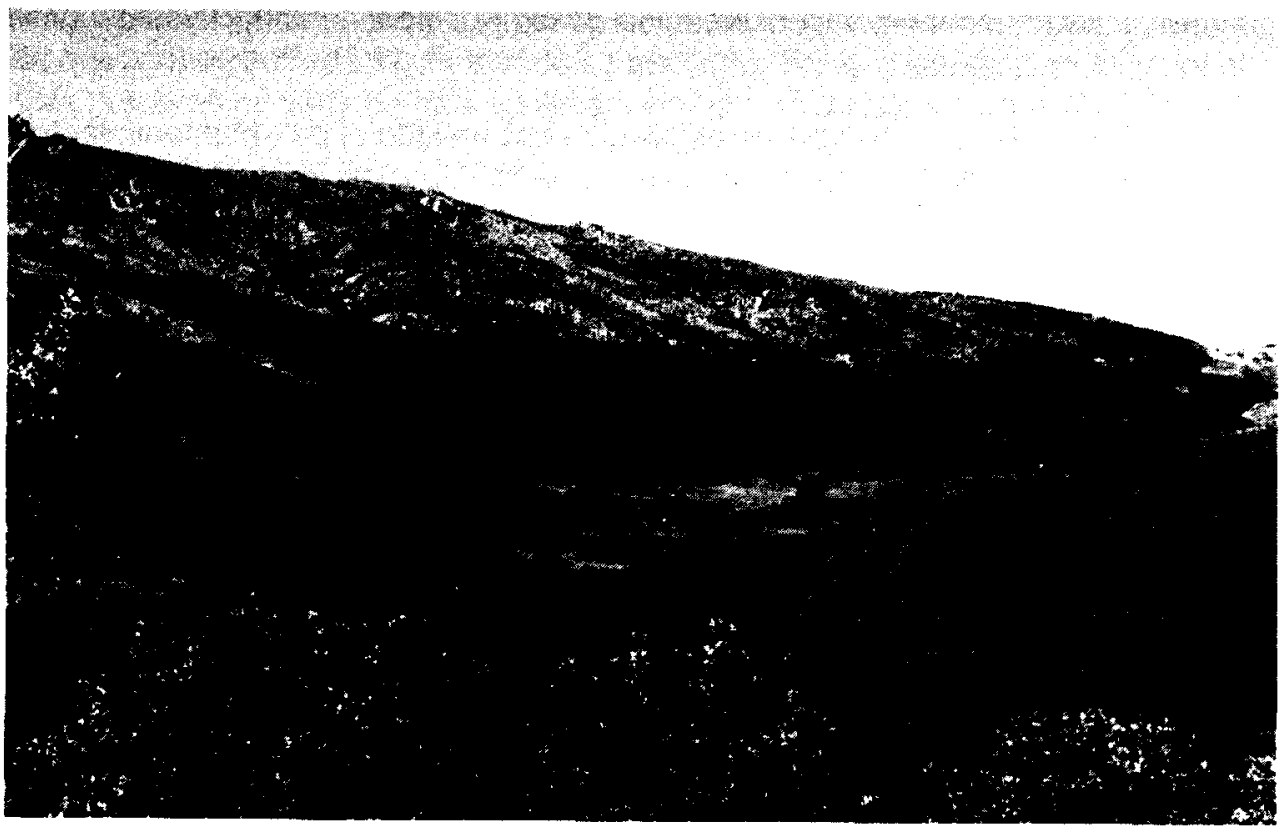

Figura 2. Puerto de la Morcuera. Variabilidad cromática debida a la vegetación.

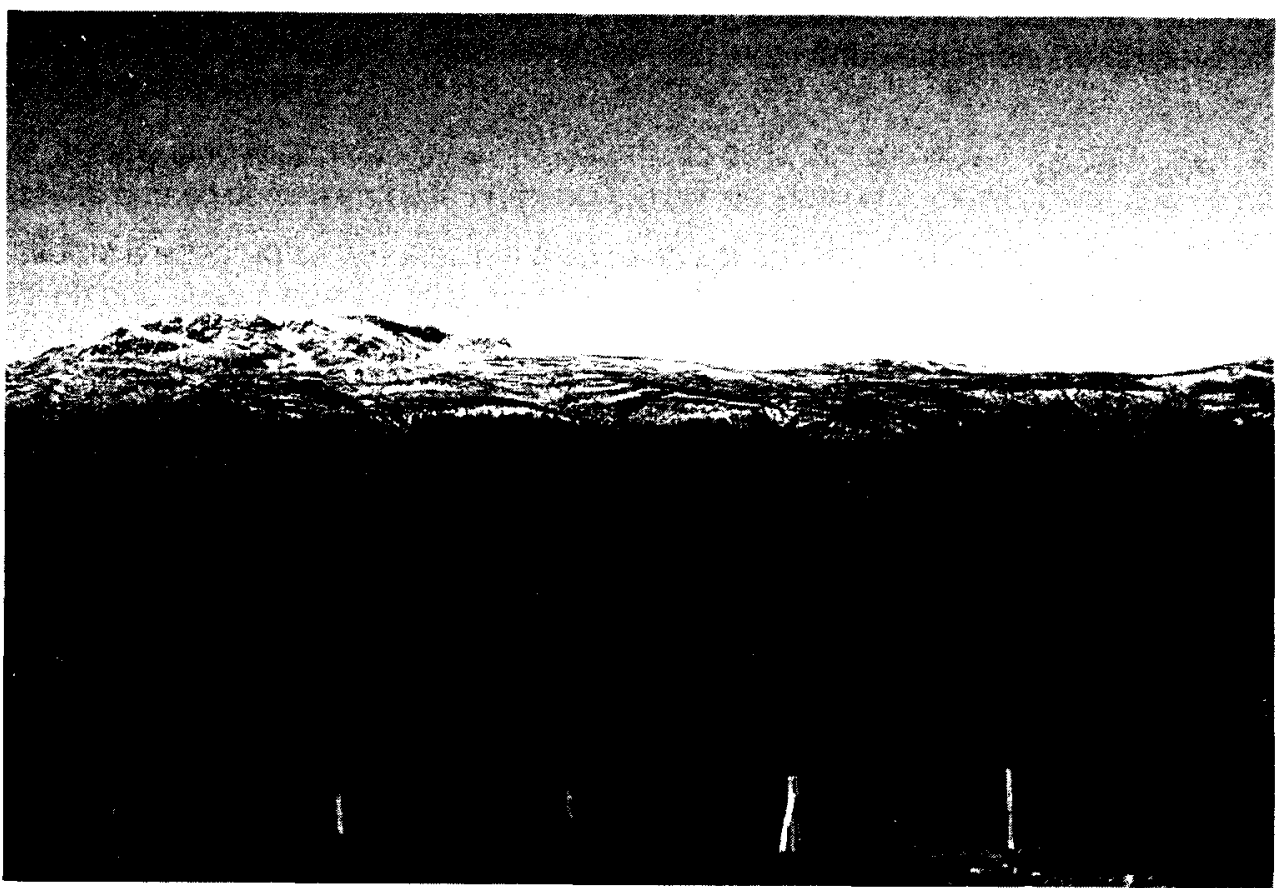

Figura 3. Macizo de Peñalara. Variabilidad cromática debida a la altitud. 
Criterio 2. Mantenimiento y Conservación de las Funciones Productivas de los Ecosistemas.

El segundo de los criterios que se ha utilizado ha sido el de "Mantenimiento y Conservación de las Funciones Productivas de los Ecosistemas». Para su medición se han considerado dos indicadores: la accesibilidad y los núcleos urbanos.

El primero de ellos se seleccionó en base a que para la realización de trabajos forestales, la defensa del monte, o "la posibilidad de disfrute" de los distintos parajes de una Comunidad, por parte de los ciudadanos, es necesaria la existencia de vías de acceso.

El segundo de los indicadores, se consideró, teniendo en cuenta los servicios que los montes producen, de los que son beneficiarios la sociedad y el entorno próximo.

\section{Criterio 3. Conservación y Mantenimiento del Suelo y del Agua como Recursos}

Por último, el tercer criterio que se ha considerado ha sido la Conservación y Mantenimiento del suelo y del agua como recursos. Para este caso el indicador que se ha utilizado ha sido el agua, cuya característica visual más importante es su tipología, es decir, si se trata de un río o arroyo (lineal) o si, por el contrario, son embalses o lagos (masa de agua). A la hora de evaluar si existe o no, riesgo de pérdida de sostenibilidad, el agua es un elemento muy importante a considerar al tratarse de un elemento dominante del paisaje visual.

\section{Evaluación}

Una vez se han seleccionado los criterios e indicadores se procede a su evaluación. Para llevar a cabo ésta, es preciso asignar unos valores a cada uno de los parámetros con los que se van a medir los indicadores. Para esto se definen unos criterios base de la elección.

Indicador diversidad visual: Este indicador se ha medido utilizando para ello tres parámetros.

El primero de ellos es la textura. Por textura se entiende "la agregación, de manera indiferenciada, de formas y colores que se perciben como variaciones o irregularidades de una superficie continua". Se consideran tres tipos de texturas: fina, mediana, gruesa.

Para su medición, se asignan unos valores que hacen referencia al riesgo que cada una de ellas presenta, ante la pérdida de sostenibilidad. De esta manera, se considera, que cualquier cambio en la agrupación vegetal que presente texturas finas (asociadas a mayor intensidad o continuidad de las actividades humanas), compromete la sostenibilidad visual del paisaje, y por consiguiente, tendrá un 
riesgo mayor que aquél en el que sean las gruesas las predominantes (aquellas en las que menos actividades humanas se realizan). Por este motivo, se ha asignado a las primeras un valor de tres, frente al valor uno que se le asigna a las segundas.

El segundo parámetro que se ha utilizado para medir el indicador diversidad visual ha sido la variabilidad cromática, que evalúa el grado de diversidad de la vegetación. Para poder llevar a cabo esta evaluación, se ha tenido en cuenta, principalmente, el contraste visual entre las distintas especies. Se han utilizado, como elementos para diferenciar distintas categorias, el color y los ciclos de usos de los cultivos. Se han considerado dos tipos de variabilidad cromática, la debida a la vegetación y la debida a la altitud.

Para la medición de la variabilidad cromática, debida a la vegetación, se ha considerado la presencia o no, de la misma, en las distintas unidades de paisaje.

Desde el punto de vista de la sostenibilidad, se considera que, en un paisaje que no posee variabilidad cromática, cualquier cambio tiene un efecto inmediato difícil de ocultar. De esta manera, se ha asignado un valor positivo (+1) a las unidades con formaciones vegetales que carecen de variabilidad cromática, y se ha restado el valor $(-1)$ a las unidades que sí poseen variabilidad cromática.

El tercer parámetro que se ha utilizado ha sido la altitud. El criterio que se ha seguido se basa en la presencia de nieve, que se ha considerado que se produce a partir de los $1.500 \mathrm{~m}$. El riesgo de pérdida de sostenibilidad se ha definido como mayor, cuando la presencia de nieve dura poco o es rara. A cada tesela se le ha añadido un valor negativo $(-1)$ si su altitud es mayor a la que se ha definido, $O(+1)$ si ocurre lo contrario.

El segundo Indicador que se ha estudiado es la Accesibilidad. En este caso, el criterio que se considera es la presencia, o no, de vias de comunicación, que se ha medido de acuerdo con la presencia de vías( carreteras, pistas forestales, locales, comarcales). Se ha asignado un valor negativo $(-1)$ a aquellas teselas que no presentan carreteras, y un valor positivo $(+1)$ a aquellas que sí presentan vías, ya que esto añade riesgo.

El tercer Indicador que se ha utilizado ha sido los Núcleos Urbanos (industrias, núcleos de población rural... ) Se le ha dado un valor negativo $(-1)$ a aquellas teselas que no presentan núcleos, y un valor positivo $(+1)$, a las teselas que tengan algún tipo de edificación.

Tanto en el caso de la existencia de vías, como de núcleos urbanos, existe una relación directa con la presencia humana. Esto provoca, que en todos los lugares que se encuentren próximos a un núcleo urbano o tengan accesibilidad alta, los riesgos de pérdida de sostenibilidad sean mayores que en aquellos lugares que se hallen más alejados de la sociedad.

Finalmente, el cuarto indicador, y último, que se ha estudiado, es el Agua (presencia o ausencia de masas de agua, de cualquier tipo, en el entorno visible). 
Las teselas que no presentan cursos de agua tendrán un valor positivo $(+1)$, ya que el riesgo ante la pérdida de sostenibilidad se encuentra favorecido, y será negativo $(-1)$ en las teselas que sí presenten cursos de agua.

\section{Medición}

Una vez asignados los valores, se procede a su medición. Para esto, se han elaboraron unos mapas, para lo que se utilizó, como herramienta de trabajo, el Sistema de Información Geográfica, ArcView.

\section{RESULTADOS}

Una vez medidos los indicadores, se pasa a la obtención de los resultados, para lo cual se fijan, previamente, unos umbrales de riesgo, asignando a cada tesela, un riesgo de pérdida de sostenibilidad. Los intervalos se han determinado atendiendo a los valores, máximo y mínimo, de riesgo, que puede alcanzar cada una de las teselas.

Se han agrupado en cinco intervalos de riesgo de pérdida de sostenibilidad.

Cuadro 2. Umbrales de Riesgo de Pérdida de Sostenibilidad

\begin{tabular}{l|c|c}
\hline Mayor Riesgo de Pérdida de Sostenibilidad & Muy Alto & Intervalo 8-7 \\
\hline & Alto & Intervalo 6-4 \\
\hline & Medio & Intervalo 3-1 \\
\hline Menor Riesgo de Pérdida de Sostenibilidad & Bajo & Intervalo 0-(-2) \\
\hline
\end{tabular}

En la segunda fase, se calcula el porcentaje de superficie de las teselas con igual riesgo de pérdida de sostenibilidad con relación a la superficie total, para así obtener los valores a nivel de unidad de paisaje. A continuación se ponderan los resultados según unos valores;

Cuadro 3. Factor de Ponderación y Valores obtenidos

\begin{tabular}{c|c|c}
\hline Riesgo & $\begin{array}{c}\text { Factor de } \\
\text { Ponderación }\end{array}$ & Valor \\
\hline Muy Alto & 5 & $>420$ \\
\hline Alto & 4 & $420-340$ \\
\hline Medio & 3 & $340-260$ \\
\hline Bajo & 2 & $260-180$ \\
\hline Muy Bajo & 1 & $180-100$ \\
\hline
\end{tabular}


Los valores obtenidos oscilan entre, cuatrocientos veinte para las teselas con mayor riesgo de pérdida de sostenibilidad y cien, para las del riesgo más bajo.

Estos valores permiten elaborar los Mapas de Riesgo de Pérdida de Sostenibilidad.

A modo de ejemplo se muestran los valores obtenido para la Unidad de Puerto de Reventón (U.P. N. ${ }^{\circ}$ ).

Cuadro 4. Resultados de la Unidad de Paisaje Puerto del Reventón

\begin{tabular}{c|c|c|c|c|c}
\hline $\begin{array}{c}\text { N. }{ }^{\circ} \text { de Teselas } \\
\text { de la U.P. 4 }\end{array}$ & $\begin{array}{c}\text { Superficie } \\
\left(m^{2}\right)\end{array}$ & $\begin{array}{c}\text { \% de } \\
\text { Superficie }\end{array}$ & $\begin{array}{c}\text { Riesgo de } \\
\text { Pérdida de } \\
\text { Sostenibilidad }\end{array}$ & $\begin{array}{c}\text { Factor } \\
\text { de } \\
\text { Ponderación }\end{array}$ & Valor \\
\hline$X$ & 0 & 0 & Muy Alto & 5 & 0 \\
\hline$X$ & 0 & 0 & Alto & 4 & 0 \\
\hline 12,14 & 73.348 & 0.66 & Medio & 3 & 1.99 \\
\hline $1,2,3,5,6,9,10,11,13,15$ & 6.098 .021 & 55.22 & Bajo & 2 & 110.45 \\
\hline $4,7,8$ & 4.870 .894 & 44.11 & Muy Bajo & 1 & 44.11 \\
\hline
\end{tabular}

Estos valores han sido los utilizados para elaborar el Mapa de Riesgo de Pérdida de sostenibilidad, que se muestra a continuación.

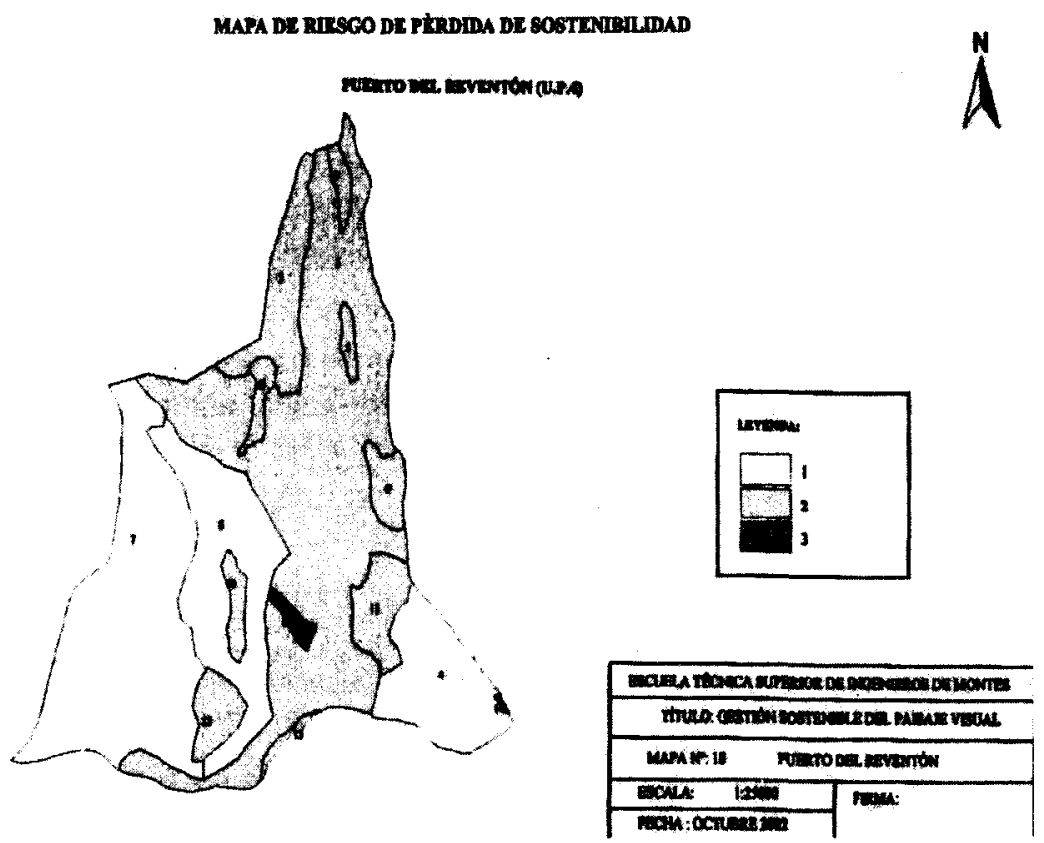

Figura 4. Mapa de Riesgo de Pérdida de Sostenibilidad. 


\section{CONCLUSIONES}

A partir del trabajo realizado sobre una zona concreta de la Comunidad de Madrid, se ha logrado definir una serie de criterios e indicadores, que nos sirven de herramienta para evaluar los riesgos de pérdida de sostenibilidad del paisaje visual.

La evaluación de Criterios e Indicadores para la Gestión Sostenible del Paisaje Visual, permite obtener una serie de conclusiones.

Con este estudio se ha elaborado un modelo, que resulta novedoso, con el que poder evaluar el Riesgo de Pérdida de Sostenibilidad Visual de un Paisaje. Como herramienta para ello se han propuesto una serie de Criterios e Indicadores, la Diversidad visual, medida mediante la textura y variabilidad cromática, la Accesibilidad, la existencia de Núcleos Urbanos y el Agua.

El relieve, a pesar de ser un elemento principal del paisaje, no se ha considerado como un indicador de manera directa. Sin embargo, se ha considerado su influencia en el paisaje indirectamente, ya que el resto de los indicadores anteriormente citados guarda relación con éste.

En el caso de la Comunidad de Madrid, donde se ha aplicado este estudio, se han obtenido resultados que demuestran, que en la Sierra Norte de Madrid, donde los valores de calidad visual son altos, el Riesgo de Pérdida de Sostenibilidad es bajo o muy bajo. Por consiguiente, se puede concluir, que son escasos los inconvenientes para que sus recursos puedan servir para satisfacer las necesidades de las generaciones futuras. Dicho de otro modo, la sostenibilidad de la zona considerada es elevada en el momento de realizar el análisis.

La información resultante de este estudio complementa a la obtenida para las distintas unidades de paisaje en un proyecto llevado a cabo por la Unidad Docente de Planificación y Proyectos de la E.T.S.I. Montes de Madrid, mediante el cual, se asignaron valores de calidad y fragilidad visual a las Unidades de Paisaje de la Comunidad de Madrid.

Dado que los criterios e indicadores utilizados se ha demostrado que son válidos, podremos extrapolar el modelo, contribuyendo así al estudio sobre la gestión de un territorio.

\section{BIBLIOGRAFÍA}

Aramburu, M. P., Escribano, R., Álvarez, S. B. y Rubio, R. (1999): Cartografía del Paisaje de la Comunidad de Madrid. Memoria. Tomo I. Madrid. Consejería de Medio Ambiente, Comunidad de Madrid y Unidad Docente de Planificación y Proyectos, Departamento de Proyectos y Planificación Rural, E.T.S.I. Montes, Universidad Politécnica de Madrid.

Aramburu, M. P., Escribano, R. y Rubio, R. (2002): Cartografía del Paisaje de la Comunidad de Madrid. Madrid. Comunidad de Madrid.

Ayuga, F. (2001): Gestión Sostenible de Paisajes Rurales. Técnicas e Ingeniería. Madrid. Fundación Alonso Martín Escudero. 
BARTHOD, C. (1998): “Criterios e indicadores de la ordenación sostenible de los bosques templados: el período 1992-1996». Unasilva 192, pp. 53-56.

Bolos, M (1992): Manual de Ciencia del Paisaje. Teoria, métodos y aplicaciones. Masson. Barcelona.

CANADIAN FOREST SERVICE (1995): The Montreal Process. Criteria and Indicators for the Conservation and Sustainable Management of Temperate and Boreal Forest. Quebec.

Escribano, R. M. ${ }^{a}$, De Frutos, M., Iglesias, E., Mataix, C. y Tofirecilla, I. (1987): El Paisaje. Ministerio de Obras Públicas y Urbanismo. Unidades Temáticas Ambientales de la Dirección General del Medio Ambiente. Madrid.

GonzÁlez Bernáldez, F (1981): Ecología del Paisaje. H. Blume Ediciones. Madrid.

GonzÁlez Echeverria, R. (2000): La Fotografia: Elemento para el Análisis y la Simulación del Paisaje Forestal. Tesis Doctoral. Escuela Técnica Superior de Ingenieros de Montes. Universidad Politécnica de Madrid. Madrid.

MEADOWS, D. «Indicators and information systems for sustainable developement». The Sustainability Institute.

MOFFAT, I. (1994): “On measuring sustainable development indicators». International Journal of Sustainable Development and World Ecology 1 (2), pp. 97-109.

Ramos, A. (1980): El Estudio del Paisaje. Cátedra de Planificación y Proyectos. E.T.S.I. Montes. U.PM. Madrid.

Ramos, A y Sotelo (1987): Paisaje Natural en la Naturaleza de Madrid. Consejeria de Agricultura. Comunidad de Madrid., pp. 231-250.

Ruiz, J. P. y GONZÁLEZ BERNÁLDEZ, F. (1983): Landsacape perception, its traditional users, the ideal landsacpe of Madrid, pp. 279-297.

TÉvar SANZ, G. (1996): "La cuenca visual en el análisis del paisaje", El paisaje en el mapa. Serie geográfica. Universidad de Alcalá de Henares, pp. 99-115.

ThOMAS, W. A. (1972): Indicators of environmental quality. Plenum Press, New York.

WIJEWARDANA, D. (1998): “Criterios e indicadores para la ordenación forestal sostenible». Actualidad Forestal Tropical. Boletín de la Organización Internacional de las Maderas Tropicales para fomentar la conservación y el desarrollo sostenible de los bosques tropicales.

WWF. WORLD WIDE FUND FOR NATURE (1993): Sustainable use of Natural Resources: concepts, Issues and Criteria. Gland. Switzerland. 